\title{
Full-Wave Analysis of the Excitation of Magnetostatic-Surface Waves by a Semi-Infinite Microstrip Transducer-Theory and Experiment
}

\author{
Manuel J. Freire, Ricardo Marqués, Member, IEEE, and Francisco Medina, Senior Member, IEEE
}

\begin{abstract}
This paper presents a new method for the complete characterization of the radiation of magnetostatic-surface waves in microstrip transmission lines with longitudinal magnetization. This method is based on the analysis of the excitation of leaky modes in microstrip lines and provides both the propagation constant and the complex impedance of the microstrip. From these quantities, the radiation resistance and other relevant characteristics of the line can be directly obtained.
\end{abstract}

Index Terms-Characteristic impedance, leaky mode, magnetostatic-surface wave (MSSW).

\section{INTRODUCTION}

$\mathbf{M}$ ICROSTRIP MAGNETOSTATIC-SURFACE-WAVE (MSSW) transducers are useful in the design of delay lines, filters, and other devices [1], [2]. Although practical transducers have finite length, the analysis of the radiation of MSSW in microstrip lines of infinite length is usually considered as a reasonable approach to practical devices [3]-[8].

One of the main problems in the design of microstrip-excited MSSW transducers is the characterization of the radiation efficiency, i.e., the calculation of the input or radiation impedance. This problem has been solved in the past by calculating the average power carried away by the MSSW excited by the microstrip through the Poynting vector associated with the MSSW [3]-[5], [8]. The calculation of the Poynting vector requires the computation of the MSSW fields. To reach this goal, both a magnetostatic approach [3], [4] and a full-wave analysis [5], [8] have been reported in the literature. In [3] and [4], a uniform surface current density is assumed on the strip and analytical expressions are obtained for the MSSW fields. In [8], the fields are analytically calculated after the surface current density is numerically computed by means of a full-wave analysis using the Galerkin method.

In this paper, we develop a method to obtain the complex propagation constant and the complex characteristic impedance

Manuscript received April 23, 2002; revised September 20, 2002. This work was supported by CICYT and FEDER funds under Project TIC2001-3163.

The authors are with the Microwave Group, Department of Electronics and Electromagnetism, University of Seville, 41012 Seville, Spain.

Digital Object Identifier 10.1109/TMTT.2003.808633 a)

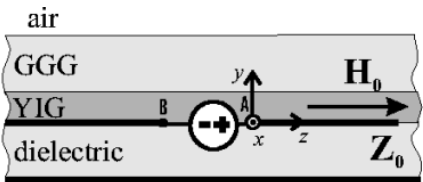

b)

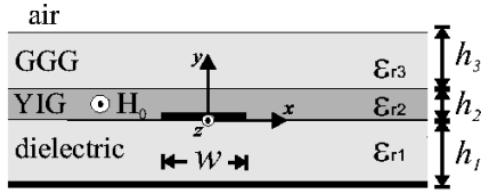

c)

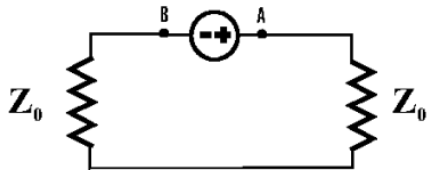

Fig. 1. (a) Two semi-infinite microstrip lines fed by a 1-V delta-gap source applied at $z=0$. (b) Cross section of the microstrip line. (c) Equivalent circuit.

of an infinitely long microstrip MSSW transducer. From these parameters, the radiation efficiency and other relevant quantities of the infinite and/or the finite length transducers are then obtained. No direct computation of the MSSW fields is required in this analysis, which is a significant advantage of the proposed method.

The following sections describe the guidelines of the method of analysis. Then, some numerical and experimental results are shown. Finally, concluding remarks are presented.

\section{Method of ANALYsis}

The method reported in [9] and [10] to analyze the excitation of leaky modes in printed-circuit lines is applied here to the analysis of the mode propagating along a YIG-loaded longitudinally magnetized microstrip line radiating MSSWs. This method consists of the full-wave analysis of the current excited in a pair of semi-infinite microstrip lines fed by a 1-V delta-gap source. Fig. 1(a) and (b) shows the structure under study. Fig. 1(c) shows the equivalent circuit of the structure.

Assuming that $\mathbf{E}^{\text {gap }}(x, z)$ is the electric field imposed by the delta-gap voltage source, the surface current density on the con- 
ductor strip $\mathbf{J}_{\mathbf{s}}$ can be obtained after solving the following electric field integral equation (EFIE) [10]:

$$
\mathbf{E}^{\text {gap }}(x, z)=\iint_{\text {strip }} \overline{\mathbf{G}}\left(x, z, x^{\prime}, z^{\prime}\right) \cdot \mathbf{J}_{s}\left(x^{\prime}, z^{\prime}\right) d x^{\prime} d z^{\prime}
$$

where $\overline{\mathbf{G}}\left(x, z, x^{\prime}, z^{\prime}\right)$ is the spatial dyadic Green's function, with the variables $x, x^{\prime}$ extended from $-w / 2$ to $w / 2$ ( $w$ stands for the strip width) and $z, z^{\prime}$ from $-\infty$ to $\infty$. The electric field in the gap is modeled as

$$
\mathbf{E}^{\text {gap }}(x, z)=\delta(z) \hat{\mathbf{z}}, \quad|x| \leq \frac{w}{2}
$$

where $\delta(z)$ is the Dirac delta function. The EFIE (1) is solved using the Galerkin method by expanding both the transverse and longitudinal components of the surface current density, $J_{x}$ and $J_{z}$, into a general set of complete domain basis functions as follows:

$$
\begin{aligned}
\mathbf{J}_{s}(x, z) & =J_{x}(x, z) \hat{\mathbf{x}}+J_{z}(x, z) \hat{\mathbf{z}} \\
J_{\alpha}(x, z) & =\sum_{n=0}^{N} F_{\alpha, n}(x) I_{\alpha, n}(z), \quad \alpha=x, z .
\end{aligned}
$$

The $I_{\alpha, n}(z)$ functions are the unknown coefficients in the Galerkin problem and the $F_{\alpha, n}(x)$ functions are the basis functions, which are given by

$$
\begin{aligned}
& F_{x, n}(x)=\frac{4}{w \pi} j U_{n}(2 x / w) \sqrt{1-\left(\frac{2 x}{w}\right)^{2}} \\
& F_{z, n}(x)=\frac{2}{w \pi} \frac{T_{n}(2 x / w)}{\sqrt{1-\left(\frac{2 x}{w}\right)^{2}}}
\end{aligned}
$$

that is, first $\left(T_{n}\right)$ and second kind $\left(U_{n}\right)$ normalized Chebyshev polynomials weighted by the proper edge condition [7], [8]. It must be noted that the integration of the $F_{z, n}(x)$ functions turns out to be

$$
\int_{-w / 2}^{w / 2} F_{z, n}(x) d x=\delta_{n 0}
$$

where $\delta_{n 0}$ is the Kronecker delta. Taking this into account, the current $I(z)$ on the strip is given by the coefficient related to the zeroth-order basis functions for $J_{z}$, that is, $I_{z, 0}(z)$. The EFIE (1) is transformed into the Fourier domain and, after applying the Galerkin method, the following matrix equation is obtained [10]:

$$
\begin{aligned}
& \sum_{\alpha, n} \Gamma_{(\beta, m),(\alpha, n)}\left(k_{z}\right) \cdot \tilde{I}_{\alpha, n}\left(k_{z}\right)=\delta_{\beta, z} \delta_{m, 0}, \\
& \alpha=x, z ; \quad \beta=x, z ; \quad m, n=0,1, \ldots, N .
\end{aligned}
$$

The $\tilde{I}_{\alpha, n}\left(k_{z}\right)$ function in (8) accounts for the Fourier transform of the unknown coefficients in (4), $\delta_{\beta, z} \delta_{m, 0}$ are the Kronecker deltas that constitute the independent elements, and $\Gamma_{(\beta, m),(\alpha, n)}\left(k_{z}\right)$ are the matrix elements given by

$$
\begin{aligned}
& \Gamma_{(\beta, m),(\alpha, n)}\left(k_{z}\right)=\frac{1}{2 \pi} \int_{C_{k_{x}}} d k_{x}[\left.\tilde{F}_{\beta, m}\left(k_{x}^{*}\right)\right]^{*} \\
& \cdot \tilde{G}_{\beta \alpha}\left(k_{x}, k_{z}\right) \tilde{F}_{\alpha, n}\left(k_{x}\right)
\end{aligned}
$$

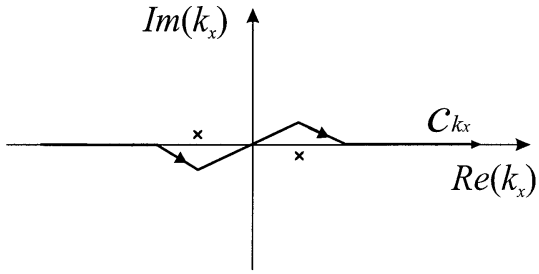

Fig. 2. Typical integration path in the complex $k_{z}$ plane. The crosses indicate the location of the poles of the SDGF.

where $\tilde{F}_{\beta, m}\left(k_{x}\right)$ and $\tilde{F}_{\alpha, n}\left(k_{x}\right)$ are the Fourier transform of the basis functions, $\tilde{G}_{\beta \alpha}\left(k_{x}, k_{z}\right)$ is the $\beta \alpha$-component of the spectral dyadic Green's function (SDGF), and $C_{k_{x}}$ is a proper integration path in the complex $k_{x}$-plane. The SDGF has poles in the complex $k_{x}$-plane and the integration path $C_{k_{x}}$ has to suitably detour around these poles [7]-[11]. The matrix equation in (8) is solved following the Kramer's rule, and the Fourier transform of the current $\tilde{I}\left(k_{z}\right)$ is finally expressed as

$$
\tilde{I}\left(k_{z}\right)=\tilde{I}_{z, 0}\left(k_{z}\right)=\frac{\operatorname{Det}[\Gamma]_{z, 0}}{\operatorname{Det}[\Gamma]}
$$

where $\operatorname{Det}[\Gamma]$ is the determinant of the $2 N \times 2 N$ Galerkin matrix (9) and $\operatorname{Det}[\Gamma]_{z, 0}$ is the determinant of the matrix obtained after substituting the column $(\beta, m)=(z, 0)$ of the matrix (9) by the column of independent terms in (8).

\section{A. Computation of the Propagation Constant}

The $\tilde{I}\left(k_{z}\right)$ function has poles in the complex $k_{z}$-plane which correspond to the propagation constants of the modes propagating along the microstrip [10]. These poles are calculated by searching for the zeros of the denominator in (10). Searching for the zeros in a structure as that shown in Fig. 1 at frequencies inside the MSSW region provides a pair of complex values, $\pm k_{z}= \pm \beta \mp j \alpha(\beta>0, \alpha>0), \beta$ being the phase constant of the mode propagating along the strip and $\alpha$ being the attenuation constant. These modal solutions correspond to a quasi-TEM mode on the microstrip line, which radiates MSSWs into the YIG slab [6], and here are obtained using the integration path, $C_{k_{x}}$, as depicted in Fig. 2.

The poles in the complex $k_{x}$ plane are located in the second and fourth quadrants, very close to the real axis. The path is deformed around this axis far from the poles to accelerate the convergence of the integration. Leaky modes in isotropic printed-circuit lines have modal solutions associated with poles that are located in the first and third quadrants in the complex $k_{x}$ plane (this means that leaky mode fields grow exponentially along the $x$ direction [11]). Since the poles in the $k_{x}$ plane associated with the mode propagating along the YIG-loaded line are located in the second and fourth quadrants, the fields of this mode decay exponentially along the $x$ direction, the imaginary part of these poles accounting for the attenuation constant in this direction. This behavior is typical of complex modes [12]. However, the SDGF poles are so close to the real axis that the exponential decay in the $x$ direction is very slow. Therefore, the reported modes would be better characterized as backward leaky modes, that is, leaky modes which radiate laterally and backward with respect to the direction of propagation of the 


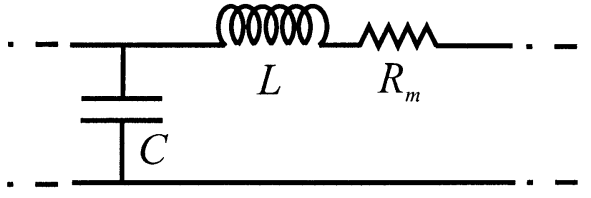

Fig. 3. Equivalent circuit in the transmission-line model of the semi-infinite YIG-loaded microstrip line. $L$ : inductance; $C$ : capacitance; $R_{m}$ : radiation resistance.

mode. This fact was already reported in [8], where it is shown that the power flux associated with the MSSW forms an angle with the strip direction greater than $90^{\circ}$.

\section{B. Computation of the Characteristic Impedance and Radiation Resistance}

As shown in [9] and [10], the residue contribution to $\tilde{I}\left(k_{z}\right)$ at the pole location given by $k_{z}=\beta-j \alpha$ in the complex $k_{z}$ plane provides the complex amplitude of the current associated with the mode of propagation constant $k_{z}$. Following this idea, the characteristic impedance of the considered microstrip mode can be readily computed. The aforementioned residue contribution is expressed as

$$
I(z)=-j \operatorname{Res}\left[\tilde{I}\left(k_{z}=\beta-j \alpha\right)\right] \exp (-j(\beta-j \alpha) z) .
$$

The residue is computed with a low numerical effort by integrating (10) around $k_{z}=\beta-j \alpha$ using a Gauss-Chebyshev quadrature, the obtained current being a complex number in general. In Fig. 1(a), the voltage imposed by the source is applied between points $A$ and $B$ being this voltage $V_{A B}=1 \mathrm{~V}$. The impedance seen between the two terminals $A$ and $B$ of the delta-gap source $Z_{A B}$ is defined as the ratio of the voltage between these points and the current at $z=0$, i.e.,

$$
Z_{A B}=\frac{V_{A B}}{I(z=0)}=\frac{V_{A B}}{-j \operatorname{Res}\left[\tilde{I}\left(k_{z}=\beta-j \alpha\right)\right]} .
$$

Fig. 1(c) shows a circuit model for the structure of Fig. 1(a). In this circuit model, two loads corresponding to the complex characteristic impedance $Z_{0}$ of the two semi-infinite lines are series connected between terminals $A$ and $B$ of the source. Therefore, the characteristic impedance $Z_{0}$ is then given by one half of the impedance between $A$ and $B$ as

$$
Z_{0}=\frac{Z_{A B}}{2}=\frac{V_{A B}}{-2 j \operatorname{Res}\left[\tilde{I}\left(k_{z}=\beta-j \alpha\right)\right]} .
$$

Thus, once the propagation constant $k_{z}=\beta-j \alpha$ is obtained, the computation of the residue of $\tilde{I}\left(k_{z}=\beta-j \alpha\right)$ provides the characteristic impedance of the line.

The radiation resistance per unit length of the line $R_{m}$ is obtained from $Z_{0}$ and $k_{z}$, using the transmission line model shown in Fig. 3, the final result being

$$
R_{m}=-\operatorname{Im}\left(Z_{0} k_{z}\right)
$$

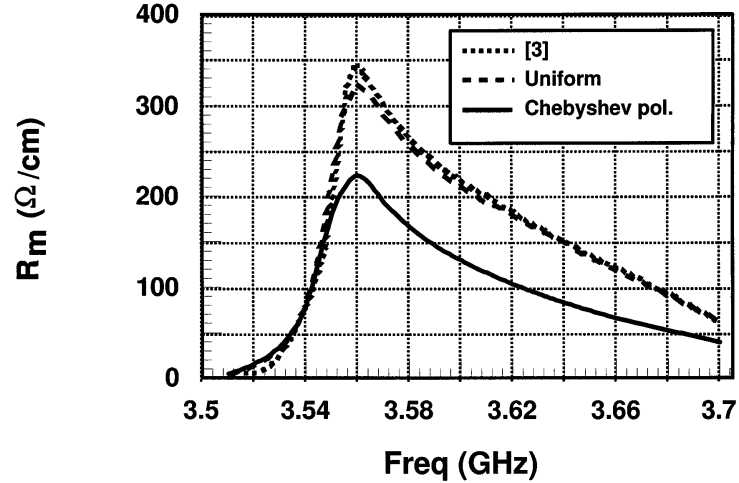

Fig. 4. Radiation resistance $R_{m}$ versus frequency. The structure is as shown in Fig. 1 with the following structural parameters: $w=178 \mu \mathrm{m}, l=2.28 \mathrm{~mm}$, $h_{1}=254 \mu \mathrm{m}, h_{2}=6.25 \mu \mathrm{m}, h_{3} \rightarrow \infty ; \varepsilon_{1}=10 \varepsilon_{0}, \varepsilon_{2}=10 \varepsilon_{0}, \varepsilon_{3}=$ $15 \varepsilon_{0} ; H_{0}=650 \mathrm{Oe}, 4 \pi M_{s}=1750 \mathrm{G}, \Delta H=0 \mathrm{Oe}$.

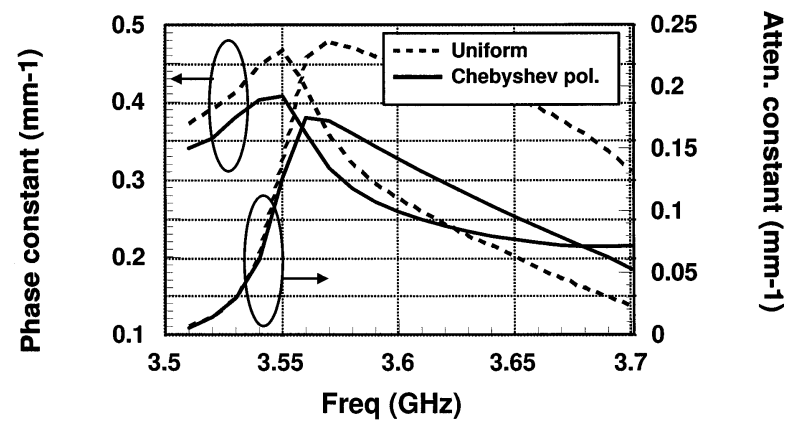

Fig. 5. Propagation constant versus frequency for the structure of Fig. 4. Results are shown for both uniform and nonuniform (Chebyshev polynomials) current distribution.

\section{NUMERICAL RESULTS}

\section{A. Theoretical Results}

In Fig. 4, our results for the radiation resistance of an infinite microstrip MSSW transducer are shown and compared with those obtained in [3]. The computation of the current has been carried out using two different choices for the basis functions. The first one consists of taking the basis functions as shown in (5) and (6), including both even and odd Chebyshev polynomials ( $N=6$ in (4) leads to the convergence of the results). The second choice consists of taking

$$
\begin{aligned}
F_{x, n}(x) & =0, \quad n \geq 0 \\
F_{z, n} & = \begin{cases}1 / w, & n=0 \\
0, & n>0\end{cases}
\end{aligned}
$$

i.e., a uniform current is imposed on the strip, as is assumed in [3]. The curves in Fig. 4 show that our computations for the radiation resistance $R_{m}$ are in agreement with those obtained in [3] following a quite different method when the same hypothesis about the distribution of the current on the strip is made. Figs. 5 and 6 show our results for the propagation constant and the complex characteristic impedance, respectively, for the same microstrip MSSW transducer of Fig. 4.

A very important conclusion concerning the modeling of practical MSSW transducers must be highlighted from these figures. Practical transducers have finite length and are 


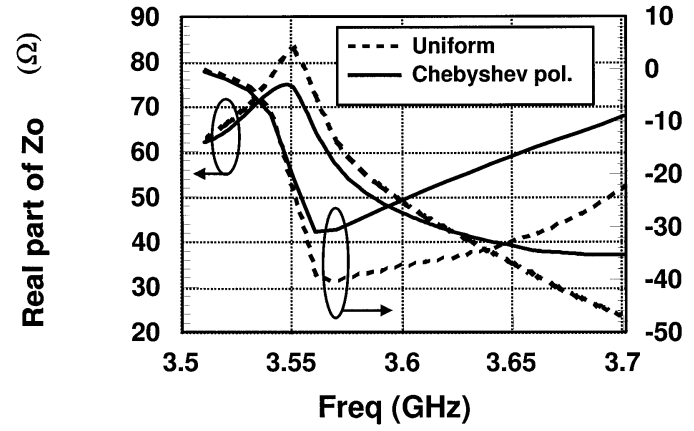

Fig. 6. Real and imaginary parts of the characteristic impedance of the structure of Fig. 4. Results are shown for both uniform and nonuniform (Chebyshev polynomials) current distribution.
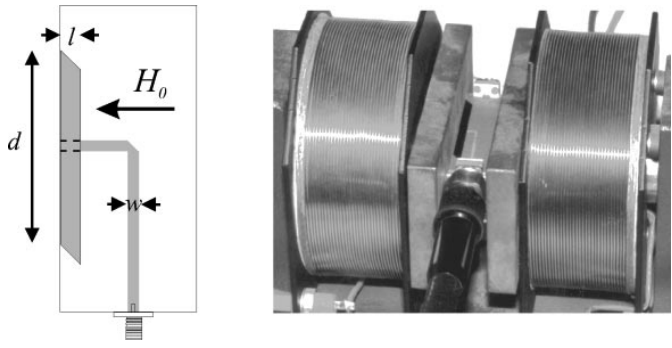

Fig. 7. Sketch of the microstrip-circuit line and picture of the experimental setup. Dimensions of the YIG sample: $d=5 \mathrm{~cm}, l=4 \mathrm{~mm}$. Strip width: $w=1.43 \mathrm{~mm}$.

short-circuited at the end [2]. Following the conventional circuit theory, the input impedance of these devices is thus given by

$$
Z_{\text {in }}=j Z_{0} \tan \left(k_{z} l\right)
$$

where $l$ is the length of the transducer. A usual approximation [3]-[5], [8] consists of assuming that $\left|k_{z} l\right| \ll 1$, so that $Z_{0} \tan \left(k_{z} l\right) \simeq Z_{0} k_{z} l$. The curves in Figs. 5 and 6 show that this assumption can be hardly justified except for very short transducers $(l \ll 1 \mathrm{~mm})$. Similar results leading to this conclusion were systematically obtained by the authors of the present paper for many other YIG-loaded microstrip configurations and static bias magnetic fields. A similar conclusion was also previously reported in [6].

\section{B. Comparison With Experimental Results}

For the purpose of verifying input impedance computations using expression (13) [and (17)], measurements have been carried out with the experimental setup shown in Fig. 7. In practical devices, the input microstrip transducer must be accompanied by an output microstrip receiver. However, the simpler microstrip-circuit line shown in Fig. 7 having a singleinput microstrip transducer fills our purpose. Input impedances for a YIG-loaded short-circuited line of finite length $l$ equal to $4 \mathrm{~mm}$ has been measured using the HP $8510 \mathrm{~B}$ automatic network analyzer. The input line is a $50-\Omega$ line built on a 0.49-mm-thick dielectric substrate, which imposes a strip width of $w=1.43 \mathrm{~mm}$ to get a $50-\Omega$ characteristic impedance. The same strip width is chosen for the YIG-loaded line section (transducer) in order to minimize transition effects. Figs. 8 and 9 shows input resistance and input reactance versus frequency. The theoretical results have been obtained from (13) and (17).

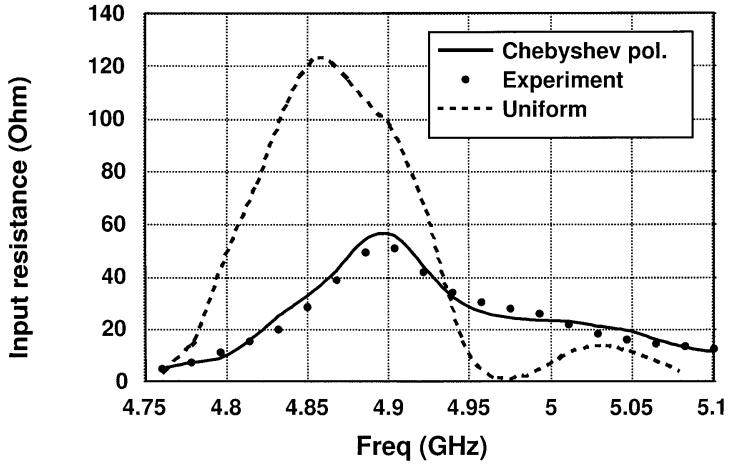

Fig. 8. Input resistance versus frequency. The structure is as shown in Fig. 1 with the following structural parameters: $w=1.43 \mathrm{~mm}, l=4 \mathrm{~mm}, h_{1}=$ $0.49 \mathrm{~mm}, h_{2}=40 \mu \mathrm{m}, h_{3}=0.5 \mathrm{~mm} ; \varepsilon_{1}=2.43, \varepsilon_{2}=10, \varepsilon_{3}=15 ; H_{0}=$ $1000 \mathrm{Oe}, 4 \pi M_{s}=1880 \mathrm{G}, \Delta H=0.6 \mathrm{Oe}$.

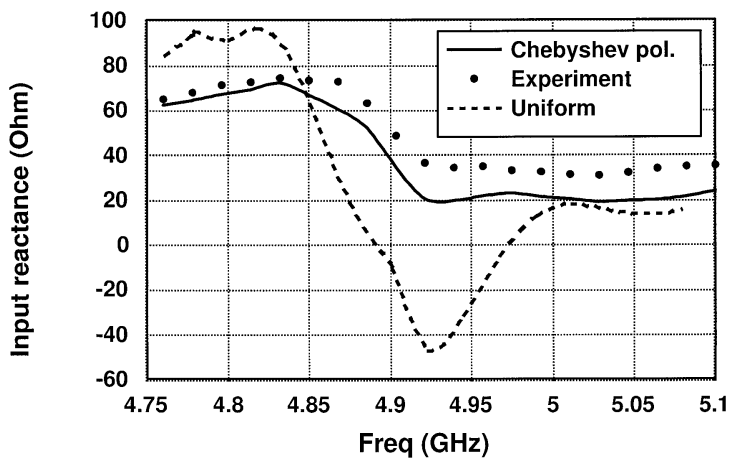

Fig. 9. Input reactance versus frequency for the structure of Fig. 8.

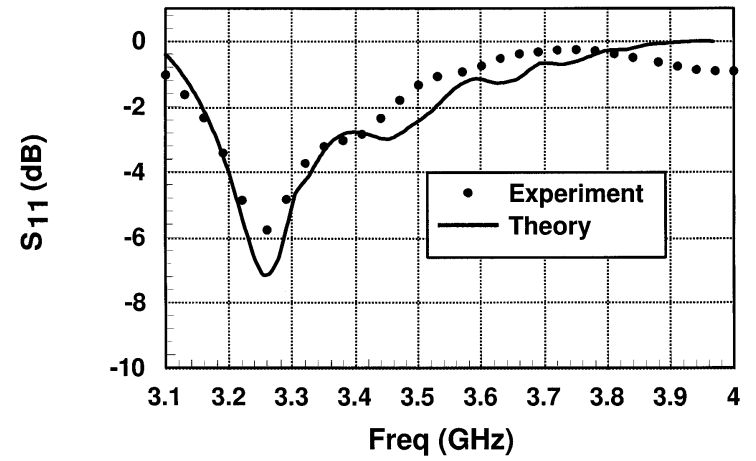

Fig. 10. Return loss in decibels. The structure is as shown in Fig. 1 with the following structural parameters: $w=1.43 \mathrm{~mm}, l=4 \mathrm{~mm}, h_{1}=0.49 \mathrm{~mm}$, $h_{2}=40 \mu \mathrm{m}, h_{3}=0.5 \mathrm{~mm} ; \varepsilon_{1}=2.43, \varepsilon_{2}=10, \varepsilon_{3}=15 ; H_{0}=500 \mathrm{Oe}$, $4 \pi M_{s}=1880 \mathrm{G}$.

The computation of the current has been carried out using two choices for the basis functions in the same way as in Fig. 4, that is, imposing a uniform current on the strip by means of (15) and (16) and using the basis functions given in (5) and (6) including both even and odd Chebyshev polynomials [with $N=6$ in (4)]. The theoretical results corresponding to the expansion into Chebyshev polynomials show a good agreement with the experimental results, which indicates that the expansion into Chebyshev polynomials seems a more realistic hypothesis than assuming a uniform current density on the strip.

Finally, Fig. 10 shows the return loss for the structure analyzed in Figs. 8 and 9 for a different bias magnetic field. A good agreement is found again between theoretical and experimental 
results. Small differences can be attributed to the finite dimensions of the YIG slab [13] and higher order modes generated at the transition between the YIG-loaded section and the dielectric-loaded section.

\section{CONCLUSION}

A new method for the complete characterization of infinitely long microstrip MSSW transducers is presented. This method is based on the analysis of the excitation of leaky modes in microstrip lines. A significant advantage of this method is that no explicit computation of fields and Poynting vector are required to obtain the line impedance and/or the radiation resistance of the transducer. The method can be applied to obtain the radiation efficiency of practical transducers of finite length by using conventional transmission-line theory. A good agreement with previous theoretical results and with experiments has been found.

\section{REFERENCES}

[1] W. S. Ishak, "Magnetostatic wave technology: A review," Proc. IEEE, vol. 76, pp. 171-187, Feb. 1988.

[2] P. Kabos and V. S. Stalmachov, Magnetostatic Waves and Their Applications. London, U.K.: Chapman \& Hall, 1994.

[3] A. K. Ganguly and D. C. Webb, "Microstrip excitation of magnetostatic surface waves: Theory and experiment," IEEE Trans. Microwave Theory Tech., vol. MTT-23, pp. 998-1006, Dec. 1975.

[4] A. K. Ganguly, D. C. Webb, and C. Banks, "Complex radiation impedance of microstrip-excited magnetostatic-surface waves," IEEE Trans. Microwave Theory Tech., vol. MTT-26, pp. 444-447, June 1978.

[5] J. C. Sethares, "Magnetostatic surface-wave transducers," IEEE Trans Microwave Theory Tech., vol. MTT-27, pp. 902-909, Nov. 1979.

[6] G. A. Vugalter and I. A. Gilinski, "Excitation and recption of magnetostatic waves by a microstrip transducer," Sov. Phys. Tech. Phys., vol. 30, no. 11, pp. 1332-1334, Nov. 1985.

[7] E.-B. El-Sharawy and R. W. Jackson, "Full-wave analysis of an infinitely long magnetic surface wave transducer," IEEE Trans. Microwave Theory Tech., vol. 38, pp. 730-738, June 1990

[8] J.-H. Lee and J.-W. Ra, "Full-wave calculation of the radiation impedance of microstrip-excited magnetic surface waves," Microwave Opt. Technol. Lett., vol. 6, no. 7, pp. 441-444, June 1993.

[9] C. Di Nallo, F. Mesa, and D. R. Jackson, "Excitation of leaky modes on multilayer stripline structures," IEEE Trans. Microwave Theory Tech. vol. 46, pp. 1062-1071, Aug. 1998.

[10] D. R. Jackson, F. Mesa, M. J. Freire, D. P. Nyquist, and C. Di Nallo, "An excitation theory for bound modes, leaky modes, and residual-wave currents on astripline structures," Radio Sci., vol. 35, no. 2, pp. 495-510, Mar. 2000.

[11] F. Mesa and R. Marqués, "Integral representation of spatial Green's function and spectral domain analysis of leaky covered strip-like lines," IEEE Trans. Microwave Theory Tech., vol. 43, pp. 828-837, Apr. 1995.

[12] M. Mrozowski, Guided Electomagnetic Waves. Somerset, U.K.: Research Studies Press, 1997

[13] T. W. O'Keeffe and R. W. Patterson, "Magnetostatic surface-wave propagation in finite samples," J. Appl. Phys., vol. 49, no. 9, pp. 4886-4895, Sept. 1978.

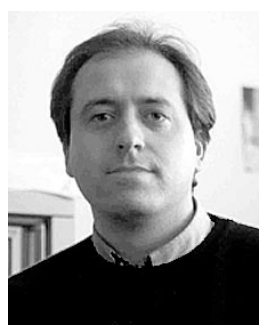

Manuel J. Freire was born in Cádiz, Spain, in 1972. He received the Licenciado and Ph.D. degrees in physics from the University of Seville, Seville, Spain, in 1995 and 2000, respectively.

From 1995 to 1996, he served in the Royal Naval Observatory, Spanish Navy, San Fernando, Cádiz, Spain. In 1996, he joined the Microwave Group, Department of Electronics and Electromagnetics, University of Seville. In 1998, he was a Visiting Researcher with the Department of Electrical and Computer Engineering, University of Houston, Houston, TX. He is currently an Assistant Professor with the Department of Electronics and Electromagnetics, University of Seville. His research interest include leakage in microwave integrated circuits, microwave propagation in ferrite devices, and periodic structures.

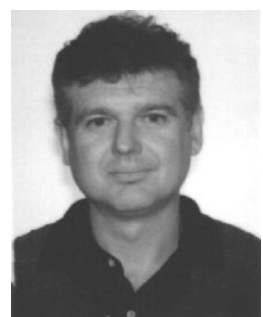

Ricardo Marqués (M'95) was born in San Fernando, Cádiz, Spain. He received the Licenciado and Doctor degrees from the University of Seville, Seville, Spain, in 1983 and 1987, respectively, both in physics.

Since 1984, he has been with the Department of Electronics and Electromagnetism, University of Seville, where he is currently an Associate Professor. His main fields of interest include computer-aided design (CAD) for microwave integrated-circuit (MIC) devices, wave propagation in ferrites, and other complex anisotropic and/or bi-isotropic media

and field theory.

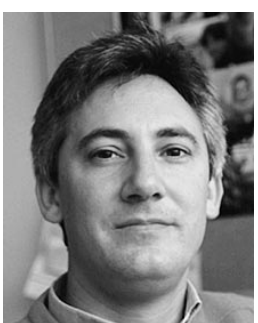

Francisco Medina (M'90-SM'01) was born in Puerto Real, Cádiz, Spain, in November 1960. He received the Licenciado and Doctor degrees from the University of Seville, Seville, Spain, in 1983 and 1987, respectively, both in physics.

From 1986 to 1987 , he spent the academic year with the Laboratoire de Microondes de l'ENSEEIHT, Toulouse, France. From 1985 to 1989 , he was a Profesor Ayudante (Assistant Professor) with the Department of Electronics and Electromagnetism, University of Seville, and since 1990, he has been a Profesor Titular (Associate Professor) of electromagnetism. He is also currently Head of the Microwaves Group, University of Seville. His research interest includes analytical and numerical methods for guidance, resonant and radiating structures, passive planar circuits, and the influence on these circuits of anisotropic materials.

Dr. Medina was a member of both the Technical Program Committee (TPC) of the 23rd European Microwave Conference, Madrid, Spain, 1993, and the TPC of ISRAMT'99, Malaga, Spain. He is on the Editorial Board of the IEEE TRANSACTIONS ON MiCROWAVE THEORY AND TECHNIQUES. He has been a reviewer for other IEEE and Institution of Electrical Engineers (IEE), U.K., publications. 